\title{
SIGNAL INPAINTING ON GRAPHS VIA TOTAL VARIATION MINIMIZATION
}

\author{
Siheng Chen ${ }^{1,2}$, Aliaksei Sandryhaila ${ }^{1}$, George Lederman ${ }^{4}$, Zihao Wang $^{4}$, José M. F. Moura ${ }^{1}$, \\ Piervincenzo Rizzo ${ }^{5}$, Jacobo Bielak ${ }^{4}$, James H. Garrett ${ }^{4}$ and Jelena Kovačević ${ }^{1,3,2}$ \\ ${ }^{1}$ Dept. of ECE, ${ }^{2}$ Center for Bioimage Informatics, ${ }^{3}$ Dept. of BME, ${ }^{4}$ Dept. of CEE, \\ Carnegie Mellon University, \\ ${ }^{5}$ Dept. of CEE, University of Pittsburgh \\ Pittsburgh, PA, USA
}

\begin{abstract}
We propose a novel recovery algorithm for signals with complex, irregular structure that is commonly represented by graphs. Our approach is a generalization of the signal inpainting technique from classical signal processing. We formulate corresponding minimization problems and demonstrate that in many cases they have closed-form solutions. We discuss a relation of the proposed approach to regression, provide an upper bound on the error for our algorithm and compare the proposed technique with other existing algorithms on realworld datasets.
\end{abstract}

Index Terms - Signal processing on graphs, signal inpainting, total variation, semi-supervised learning.

\section{INTRODUCTION}

The problem of collecting, processing and analyzing data obtained from or represented by networks has been receiving a constantly increasing interest due to the abundance of such data in various research fields. An integral part of solving this problem is the development of new models and techniques that can be applied to datasets with complex irregular structures.

Recently, a theoretical framework called signal processing on graphs has emerged as a new approach to analyze signals with irregular structure $[1,2,3,4]$. Its key idea is to represent the structure of a signal with a graph by associating signal coefficients with graph nodes and analyzing graph signals by using appropriately defined signal processing techniques, such as Fourier transform, filtering, and wavelets.

In this paper, we study the problem of signal recovery, that is, reconstruction or estimation of signal coefficients that are missing, unmeasurable, or corrupted by noise, a task often referred to as signal inpainting [5, 6, 7].

Previous work on this topic has primarily come when missing signal coefficients are unknown labels that need to be learned [8, 9]. Existing approaches are often based on the graph Laplacian operator and take roots in spectral graph theory [10]. From the perspective of signal processing, they

The authors gratefully acknowledge support from the NSF through awards 1130616 and 1017278, and CMU Carnegie Institute of Technology Infrastructure Award. seek to minimize a total variation function based on the graph Laplacian, which measures how much signal coefficients differ from other relevant coefficients. A major limitation of the graph Laplacian based method is its restriction to undirected graphs with real, non-negative edge weights.

We propose a novel approach to graph signal inpainting. It seeks to minimizes a total variation function that is based on a graph shift, which is a fundamental signal processing concept that can be defined for signals represented by any graph $[1,2]$. As a result, our proposed technique is applicable to a much broader class of graphs. In this paper, we offer two formulations of the signal inpainting on graphs as minimization problems and demonstrate that in many cases these problems have exact closed-form solutions. We also discuss a connection of our approach to regression on graphs, derive an upper bound on the signal inpainting error produced by our technique, and demonstrate on real-world datasets that our approach can lead to better signal recovery and signal classification than other existing approaches.

\section{DISCRETE SIGNAL PROCESSING ON GRAPHS}

In this section, we briefly review relevant concepts of discrete signal processing on graphs; a thorough introduction can be found in $[1,2]$. Discrete signal processing on graphs is a theoretical framework that generalizes classical discrete signal processing from regular domains, such as lines and rectangular lattices, to irregular structures that are commonly described by graphs.

Consider a graph $G=(\mathcal{V}, \mathrm{A})$, where $\mathcal{V}=\left\{v_{n}\right\}_{n=1}^{N}$ is the set of nodes and $\mathrm{A} \in \mathbb{C}^{N \times N}$ is a graph shift, or an adjacency matrix, that is, an elementary filtering operation that replaces a signal coefficient at a node with a weighted linear combination of coefficients at its neighboring nodes. Each edge weight $\mathrm{A}_{i, j}$ characterizes the relation between the $i$ th node and the $j$ th node. For example, they can quantify similarities and dependencies between nodes, or indicate communication patterns in networks. The graph shift operation is written as

$$
\mathrm{x} \rightarrow \mathrm{A} \mathbf{x} .
$$

where a graph signal $\mathbf{x}$ is defined as a mapping

$$
\mathbf{x}: \mathcal{V} \rightarrow \mathbb{C}^{n}
$$


that assigns a signal coefficient $x_{n} \in \mathbb{C}$ to the graph node $v_{n}$.

Smoothness of graph signals is expressed by a graph total variation function

$$
\mathrm{TV}_{\mathrm{A}}(\mathbf{x})=\left\|\mathbf{x}-\frac{1}{\left|\lambda_{\max }(\mathrm{A})\right|} \mathrm{A} \mathbf{x}\right\|_{1},
$$

where $\lambda_{\max }(\mathrm{A})$ denote the eigenvalue of $\mathrm{A}$ with the largest magnitude. For notational simplicity, assume that graph shift A has been normalized to satisfy $\lambda_{\max }(\mathrm{A})=1$. Note that when the graph shift $\mathrm{A}$ is the cyclic permutation matrix, representing time-series signals, (1) follows the same definition in classical signal processing. We define the quadratic form of graph total variation as follows:

$$
\mathrm{S}_{2}(\mathbf{x})=\frac{1}{2}\|\mathbf{x}-\mathrm{A} \mathbf{x}\|_{2}^{2} .
$$

Since a quadratic form is easy for computation, we use (2) to measure the smoothness of a graph signal in this paper.

\section{SIGNAL INPAINTING ON GRAPHS}

Signal inpainting is a process of recovering missing or corrupted signal coefficients from a known part of the signal. In discrete-time signal processing, a signal, such as a time series or a digital image, is typically assumed to be smooth, and the missing part is recovered through regularization or lowpass filtering $[5,6,7]$.

\subsection{Problem formulation}

The graph total variation (1) allows us to generalize the inpainting process from lines and rectangular lattices to arbitrary graphs. We work with graph signals of the form

$$
\mathbf{x}=\left[\begin{array}{c}
\mathbf{x}_{\mathcal{M}} \\
\mathbf{x}_{\mathcal{U}}
\end{array}\right]
$$

where $\mathbf{x}_{\mathcal{M}} \in \mathbb{C}^{M}$ is the known part of the signal and $\mathbf{x}_{\mathcal{U}} \in$ $\mathbb{C}^{N-M}$ is the unknown part (without loss of generality, we assume that the known coefficients $x_{1}, \ldots, x_{M}$ correspond to the first $M$ graph nodes $v_{1}, \ldots, v_{M}$; this arrangement can always be achieved by reordering nodes). Assuming that (3) is a smooth signal, that is, its variation is small, we recover the missing part $\mathbf{x}_{\mathcal{U}}$ by solving the following minimization problem:

$$
\begin{aligned}
\mathbf{x}^{*}= & \operatorname{argmin} \mathrm{S}_{2}(\widehat{\mathbf{x}}), \\
\text { subject to } & \left\|\widehat{\mathbf{x}}_{\mathcal{M}}-\mathbf{x}_{\mathcal{M}}\right\|_{2}^{2} \leq \epsilon^{2} .
\end{aligned}
$$

The condition (4b) controls how well the known part of the signal is preserved.

\subsection{Graph total variation regularization}

Alternatively, the graph signal inpainting (4) can be formulated as an unconstrained problem,

$$
\mathbf{x}^{*}=\operatorname{argmin} \frac{1}{2}\left\|\widehat{\mathbf{x}}_{\mathcal{M}}-\mathbf{x}_{\mathcal{M}}\right\|_{2}^{2}+\lambda \mathrm{S}_{2}(\widehat{\mathbf{x}}),
$$

where the tuning parameter $\lambda$ controls the trade-off between two parts of the objective function. Equation (5) is called as graph total variation regularization (GTVR). Small values of $\lambda$ emphasize fitting the estimates to the known measurements more than the smoothness on the graph; large values of $\lambda$ lead to smoother solutions of (5).

Note that (5) is a convex quadratic problem and has a closed-form solution. For many values of $\epsilon$ in (4) we can find a corresponding value of $\lambda$ for which (5) yields an equivalent solution.

We now derive a closed-form solution to (5). For notational simplicity, assume that matrix $\mathrm{A}$ has been normalized to satisfy $\lambda_{\max }(\mathrm{A})=1$, so we can write the variation function (1) as

$$
\begin{aligned}
\mathrm{S}_{2}(\mathbf{x}) & =\frac{1}{2}\|\mathbf{x}-\mathrm{A} \mathbf{x}\|_{2}^{2} \\
& =\frac{1}{2} \mathbf{x}^{H}(\mathrm{I}-\mathrm{A})^{H}(\mathrm{I}-\mathrm{A}) \mathbf{x}, \\
& =\frac{1}{2} \mathbf{x}^{H} \widetilde{\mathrm{A}} \mathbf{x},
\end{aligned}
$$

where $\mathrm{I}$ is the identity matrix and $\widetilde{\mathrm{A}}=(\mathrm{I}-\mathrm{A})^{H}(\mathrm{I}-\mathrm{A})$. The objective function in (5) is thus a linear combination of two quadratic functions of $\widehat{\mathbf{x}}$. The derivative of the objective function in (5) is:

$$
\begin{aligned}
& \frac{\partial}{\partial \widehat{\mathbf{x}}}\left(\frac{1}{2}\left\|\widehat{\mathbf{x}}_{\mathcal{M}}-\mathbf{x}_{\mathcal{M}}\right\|_{2}^{2}+\lambda \mathrm{S}_{2}(\widehat{\mathbf{x}})\right) \\
= & \frac{1}{2} \frac{\partial}{\partial \widehat{\mathbf{x}}}\left((\widehat{\mathbf{x}}-\mathbf{x})^{H}\left[\begin{array}{cr}
\mathrm{I}_{M} & \mathbf{0} \\
\mathbf{0} & \mathbf{0}
\end{array}\right](\widehat{\mathbf{x}}-\mathbf{x})+\lambda \widehat{\mathbf{x}}^{H} \widetilde{\mathrm{A}} \widehat{\mathbf{x}}\right) \\
= & {\left[\begin{array}{cc}
\mathrm{I}_{M} & \mathbf{0} \\
\mathbf{0} & \mathbf{0}
\end{array}\right](\widehat{\mathbf{x}}-\mathbf{x})+\lambda \widetilde{\mathrm{A}} \widehat{\mathbf{x}} . }
\end{aligned}
$$

By setting (7) to zero, we then obtain the closed-form solution as follows:

$$
\mathbf{x}^{*}=\left(\left[\begin{array}{cc}
\mathrm{I}_{M} & \mathbf{0} \\
\mathbf{0} & \mathbf{0}
\end{array}\right]+\lambda \widetilde{\mathrm{A}}\right)^{-1}\left[\begin{array}{c}
\mathbf{x}_{\mathcal{M}} \\
\mathbf{0}
\end{array}\right] .
$$

\subsection{Graph total variation minimization}

When the known part of the signal needs to be preserved intact, we must solve (4) directly for $\epsilon=0$,

$$
\begin{aligned}
\mathrm{x}^{*}= & \operatorname{argmin} \mathrm{S}_{2}(\widehat{\mathbf{x}}), \\
\text { subject to } & \widehat{\mathbf{x}}_{\mathcal{M}}=\mathrm{x}_{\mathcal{M}} .
\end{aligned}
$$

Equation (9) is called as graph total variation minimization (GTVM). By writing $\widetilde{A}$ in a block form as

$$
\widetilde{\mathrm{A}}=\left[\begin{array}{cc}
\widetilde{\mathrm{A}}_{\mathcal{M} \mathcal{M}} & \widetilde{\mathrm{A}}_{\mathcal{M U}} \\
\widetilde{\mathrm{A}}_{\mathcal{U M}} & \widetilde{\mathrm{A}}_{\mathcal{U U}}
\end{array}\right],
$$


we can rewrite the objective function in (9) as

$$
\begin{aligned}
\mathrm{S}_{2}(\widehat{\mathbf{x}})= & \frac{1}{2} \widehat{\mathbf{x}}^{H} \widetilde{\mathrm{A}} \widehat{\mathbf{x}} \\
= & \frac{1}{2}\left[\begin{array}{ll}
\widehat{\mathbf{x}}_{\mathcal{M}}^{H} & \widehat{\mathbf{x}}_{\mathcal{U}}^{H}
\end{array}\right]\left[\begin{array}{cc}
\widetilde{\mathrm{A}}_{\mathcal{M} \mathcal{M}} & \widetilde{\mathrm{A}}_{\mathcal{M U}} \\
\widetilde{\mathrm{A}}_{\mathcal{U M}} & \widetilde{\mathrm{A}}_{\mathcal{U} \mathcal{U}}
\end{array}\right]\left[\begin{array}{ll}
\widehat{\mathbf{x}}_{\mathcal{M}}^{H} & \widehat{\mathbf{x}}_{\mathcal{U}}^{H}
\end{array}\right] \\
= & \frac{1}{2}\left(\widehat{\mathbf{x}}_{\mathcal{M}}^{H} \widetilde{\mathrm{A}}_{\mathcal{M} \mathcal{M}} \widehat{\mathbf{x}}_{\mathcal{M}}+\widehat{\mathbf{x}}_{\mathcal{U}}^{H} \widetilde{\mathrm{A}}_{\mathcal{U} \mathcal{M}} \widehat{\mathbf{x}}_{\mathcal{M}}\right. \\
& \left.+\widehat{\mathbf{x}}_{\mathcal{M}}^{H} \widetilde{\mathrm{A}}_{\mathcal{M} \mathcal{U}} \widehat{\mathbf{x}}_{\mathcal{U}}+\widehat{\mathbf{x}}_{\mathcal{U}}^{H} \widetilde{\mathrm{A}}_{\mathcal{U} \mathcal{U}} \widehat{\mathbf{x}}_{\mathcal{U}}\right) .
\end{aligned}
$$

Since $\widehat{\mathbf{x}}_{\mathcal{M}}=\mathbf{x}_{\mathcal{M}}$, as specified by (9b), the minimum of the objective function (11) is found by setting its derivative to zero, which yields the closed-form solution

$$
\widehat{\mathbf{x}}_{\mathcal{U}}=-\widetilde{\mathrm{A}}_{\mathcal{U} \mathcal{U}}^{-1} \widetilde{\mathrm{A}}_{\mathcal{U M}} \mathbf{x}_{\mathcal{M}}
$$

\section{DISCUSSION}

We now discuss several properties of our proposed signal inpainting algorithms.

\subsection{Connection to Graph-based Regression}

We can construct a graph to represent a dataset and a set of labels by associating each dataset element with a node and viewing labels as a signal on this graph. In this setting, graph signal inpainting becomes analogous to a regression, since it estimates a regression function that assigns a label to each node. 8]

In general, the graph-based regression has the form $[11,9$,

$$
\mathbf{x}^{*}=\operatorname{argmin} \frac{1}{2}\left\|\widehat{\mathbf{x}}_{\mathcal{M}}-\mathbf{x}_{\mathcal{M}}\right\|^{2}+\lambda \widehat{\mathbf{x}}^{H} \mathrm{G} \widehat{\mathbf{x}}
$$

where $\mathrm{G}$ is a smoothing matrix that can be defined in different ways depending on the context. For instance, if $\mathrm{G}$ is the graph Laplacian matrix, then (13) solves Laplacian regularization [9]. If, in addition, $\widehat{\mathbf{x}}_{\mathcal{M}}=\mathbf{x}_{\mathcal{M}}$, then the minimization (13) is based on the Laplacian harmonic functions [8]. Since our approach (5) uses the total variation defined by the graph shift, we use $\mathrm{G}=(1 / 2)(\mathrm{I}-\mathrm{A})^{H}(\mathrm{I}-\mathrm{A})$ in (13).

\subsection{Error Analysis}

We now derive an upper bound on the estimation error of graph signal inpainting algorithm (4).

Let $\mathrm{x}^{0}$ be the true graph signal that we are trying to estimate by signal inpainting. Assume that $\mathrm{S}_{2}\left(\mathbf{x}^{0}\right)=\eta^{2} / 2$ and $\mathbf{x}^{0}$ satisfies (4b), so that $\left\|\mathbf{x}_{\mathcal{M}}^{0}-\mathbf{x}_{\mathcal{M}}\right\|_{2}^{2} \leq \epsilon^{2}$. Also, write A in a block form similarly to (10).

Lemma 1. The estimation error $\mathrm{w}=\mathrm{x}^{0}-\mathrm{x}^{*}$ of the signal inpainting algorithm (4) is bounded by the unmeasured part of the signal as

$$
\|\mathbf{w}\|_{2} \leq \frac{q}{2}\|\mathbf{w} \mathcal{U}\|_{2}+p|\epsilon|+|\eta|
$$

where

$$
p=\left\|\left[\begin{array}{c}
\mathrm{I}_{\mathcal{M M}}+\mathrm{A}_{\mathcal{M M}} \\
\mathrm{A}_{\mathcal{U M} \mathcal{M}}
\end{array}\right]\right\|_{2}, q=\left\|\left[\begin{array}{c}
\mathrm{A}_{\mathcal{M U}} \\
\mathrm{I}_{\mathcal{U U}}+\mathrm{A}_{\mathcal{U U}}
\end{array}\right]\right\|_{2},
$$

and $\|\cdot\|_{2}$ for matrices denotes the spectral norm.

Proof. Since $\left\|\mathbf{w}_{\mathcal{M}}\right\|_{2}=\left\|\mathbf{x}_{\mathcal{M}}^{0}-\mathbf{x}_{\mathcal{M}}^{*}\right\|_{2} \leq\left\|\mathbf{x}_{\mathcal{M}}^{0}-\mathbf{x}_{\mathcal{M}}\right\|_{2}+$ $\left\|\mathbf{x}_{\mathcal{M}}-\mathbf{x}_{\mathcal{M}}^{*}\right\|_{2}=2|\epsilon|$, we obtain

$$
\begin{aligned}
\|(\mathrm{I}+\mathrm{A}) \mathbf{w}\|_{2}= & \left\|\left[\begin{array}{cc}
\mathrm{I}_{\mathcal{M} \mathcal{M}}+\mathrm{A}_{\mathcal{M} \mathcal{M}} & \mathrm{A}_{\mathcal{M U}} \\
\mathrm{A}_{\mathcal{U M}} & \mathrm{I}_{\mathcal{U} \mathcal{U}}+\mathrm{A}_{\mathcal{U} \mathcal{U}}
\end{array}\right]\left[\begin{array}{l}
\mathbf{w}_{\mathcal{M}} \\
\mathbf{w}_{\mathcal{U}}
\end{array}\right]\right\|_{2} \\
\leq & \left\|\left[\begin{array}{c}
\mathrm{I}_{\mathcal{M} \mathcal{M}}+\mathrm{A}_{\mathcal{M} \mathcal{M}} \\
\mathrm{A}_{\mathcal{U M}}
\end{array}\right]\right\|_{2} \cdot\left\|\mathbf{w}_{\mathcal{M}}\right\|_{2} \\
& +\left\|\left[\begin{array}{c}
\mathrm{A}_{\mathcal{M U}} \\
\mathrm{I}_{\mathcal{U} \mathcal{U}}+\mathrm{A}_{\mathcal{U} \mathcal{U}}
\end{array}\right]\right\|_{2} \cdot\left\|\mathbf{w}_{\mathcal{U}}\right\|_{2} \\
= & p\left\|\mathbf{w}_{\mathcal{M}}\right\|_{2}+q\left\|\mathbf{w}_{\mathcal{U}}\right\|_{2} \\
= & 2 p|\epsilon|+q\left\|\mathbf{w}_{\mathcal{U}}\right\|_{2} .
\end{aligned}
$$

Since both $\mathrm{x}^{0}$ and $\mathrm{x}^{*}$ satisfy (4b), then $\mathrm{S}_{2}\left(\mathrm{x}^{*}\right) \leq \mathrm{S}_{2}\left(\mathrm{x}^{0}\right)$, and we obtain

$$
\begin{aligned}
\|(\mathrm{I}-\mathrm{A}) \mathbf{w}\|_{2} & =\left\|(\mathrm{I}-\mathrm{A})\left(\mathbf{x}^{0}-\mathbf{x}^{*}\right)\right\|_{2} \\
& \leq\left\|(\mathrm{I}-\mathrm{A}) \mathbf{x}^{0}\right\|_{2}+\left\|(\mathrm{I}-\mathrm{A}) \mathbf{x}^{*}\right\|_{2} \\
& \leq 2\left\|(\mathrm{I}-\mathrm{A}) \mathbf{x}^{0}\right\|_{2} \\
& \leq 2|\eta|
\end{aligned}
$$

Combining these inequalities, we get

$$
\begin{aligned}
\|\mathbf{w}\|_{2} & =\left\|\frac{1}{2}(\mathrm{I}+\mathrm{A}+\mathrm{I}-\mathrm{A}) \mathbf{w}\right\|_{2} \\
& \leq \frac{1}{2}\left(\|(\mathrm{I}+\mathrm{A}) \mathbf{w}\|_{2}+\|(\mathrm{I}-\mathrm{A}) \mathbf{w}\|_{2}\right) \\
& \leq \frac{1}{2}\left(2 p|\epsilon|+q\left\|\mathbf{w}_{\mathcal{U}}\right\|_{2}+2|\eta|\right),
\end{aligned}
$$

which yields (14).

Theorem 1. If $q<2$, then the estimation error on the unknown part is bounded by

$$
\left\|\mathbf{w}_{\mathcal{U}}\right\|_{2} \leq \frac{2 p|\epsilon|+2|\eta|}{2-q}
$$

Proof. Using Lemma 1, we have

$$
\left\|\mathbf{w}_{\mathcal{U}}\right\|_{2} \leq\|\mathbf{w}\|_{2} \leq p|\epsilon|+\frac{q}{2}\left\|\mathbf{w}_{\mathcal{U}}\right\|_{2}+|\eta|
$$

By rearranging the terms in (16), we obtain (15).

The condition $q<2$ in Theorem 1 may not hold for some matrices. However, if $\mathrm{A}$ is symmetric, we have $q \leq\|\mathrm{I}+\mathrm{A}\|_{2}$ $\leq\|\mathrm{I}\|_{2}+\|\mathrm{A}\|_{2}=2$, since $\|\mathrm{A}\|_{2}=1$. Also, note that the upper bound is related to the smoothness of the true graph signal and the noise level of the measured part. A central assumption of any inpainting technique is that the true signal $\mathbf{x}^{0}$ is smooth. If this assumption does not hold, then the upper bound is large and useless. When the noise level of the measured part is smaller, the measurements from the known part are closer to the true values, which leads to a smaller estimation error. 


\begin{tabular}{lrrrrrr}
\hline \multicolumn{1}{c}{ Method } & & \multicolumn{5}{c}{ Ratio of known labels } \\
& $\lambda$ & $0.5 \%$ & $1 \%$ & $2 \%$ & $5 \%$ & $10 \%$ \\
\hline GTVM & & 80.25 & $\mathbf{9 4 . 7 6}$ & $\mathbf{9 5 . 1 8}$ & $\mathbf{9 5 . 2 8}$ & 95.20 \\
GTVR & $\mathbf{0 . 0 1}$ & 80.16 & 94.68 & 95.09 & 95.18 & 95.13 \\
& $\mathbf{0 . 1}$ & 79.99 & 94.47 & 95.11 & 95.19 & 95.15 \\
& $\mathbf{1}$ & 75.64 & 92.62 & 94.99 & 95.26 & 95.25 \\
& $\mathbf{1 0}$ & 61.68 & 68.13 & 89.02 & 95.15 & $\mathbf{9 5 . 4 3}$ \\
& $\mathbf{1 0 0}$ & 61.59 & 57.26 & 54.72 & 73.62 & 92.28 \\
HF & & 51.58 & 53.15 & 60.75 & 86.05 & 94.68 \\
LapR & $\mathbf{0 . 0 1}$ & 51.47 & 53.48 & 60.79 & 85.90 & 94.62 \\
& $\mathbf{0 . 1}$ & 50.64 & 54.40 & 61.14 & 85.22 & 94.55 \\
& $\mathbf{1}$ & 50.25 & 56.49 & 60.50 & 79.29 & 93.94 \\
& $\mathbf{1 0}$ & 50.07 & 49.84 & 54.39 & 62.83 & 80.69 \\
& $\mathbf{1 0 0}$ & 51.29 & 49.74 & 50.16 & 52.04 & 55.95 \\
AGF & & $\mathbf{8 4 . 8 1}$ & 88.36 & 94.08 & 95.00 & 95.10 \\
\hline
\end{tabular}

Table 1: Accuracy of political blog classification.

\begin{tabular}{lrrrrrr}
\hline Method & & \multicolumn{5}{c}{ Ratio of known masses } \\
& $\lambda$ & $0.5 \%$ & $1 \%$ & $2 \%$ & $5 \%$ & $10 \%$ \\
\hline GTVM & & 20.18 & 9.67 & 5.29 & 3.93 & 3.54 \\
GTVR & $\mathbf{0 . 0 1}$ & 14.71 & 9.28 & 4.92 & 3.88 & 3.52 \\
& $\mathbf{0 . 1}$ & 12.89 & 8.97 & 4.95 & 3.92 & 3.49 \\
& $\mathbf{1}$ & 10.12 & 9.11 & 5.14 & 3.58 & 3.24 \\
& $\mathbf{1 0}$ & $\mathbf{8 . 8 4}$ & 10.49 & 4.12 & 3.08 & 2.84 \\
HF & $\mathbf{1 0 0}$ & 15.70 & $\mathbf{7 . 1 3}$ & $\mathbf{3 . 8 0}$ & $\mathbf{3 . 0 0}$ & $\mathbf{2 . 8 0}$ \\
LapR & $\mathbf{0 . 0 1}$ & 38.66 & 19.44 & 5.29 & 3.31 & 2.9 \\
& $\mathbf{0 . 1}$ & 39.52 & 19.49 & 5.30 & 3.32 & 2.9 \\
& $\mathbf{1}$ & 46.29 & 24.78 & 6.83 & 3.65 & 2.95 \\
& $\mathbf{1 0}$ & 74.38 & 52.64 & 22.90 & 9.44 & 5.13 \\
& $\mathbf{1 0 0}$ & 96.32 & 85.43 & 66.84 & 47.86 & 32.1 \\
& & & & & & \\
\hline
\end{tabular}

Table 2: The mean square error for the bridge condition identification.

\section{EXPERIMENTS}

We now apply the proposed algorithm to the classification of online blogs and to the bridge condition identification for indirect bridge structural health monitoring. We compare the proposed algorithm with Laplacian regularization (LapR) and harmonic functions (HF) discussed in Section 4.1. In classification of online blogs, we also compare the proposed algorithm with adaptive graph filtering (AGF) $[12,13]$, which is a semi-supervised classifier that combines the decisions from multiple graph filters using a semi-supervised weighting function.

\subsection{Classification of online blogs}

We consider the problem of classifying $N=1224$ online political blogs as either conservative or liberal [14]. We represent conservative labels as +1 and liberal ones as -1 .

The blogs are represented by a graph in which nodes represent blogs, and directed graph edges correspond to hyperlink references between blogs. For a node $v_{n}$ its outgoing edges have weights $1 / \operatorname{deg}\left(v_{n}\right)$, where $\operatorname{deg}\left(v_{n}\right)$ is the out-degree of $v_{n}$ (the number of outgoing edges). We randomly labeled $0.5 \%, 1 \%, 2 \%, 5 \%$ and $10 \%$ of blogs and applied the inpainting algorithms to estimate the labels for remaining nodes. Es- timated labels were thresholded around zero, so that positive values were set to +1 and negative to -1 .

Classification accuracies of GTVM, GTVR, HF, LapR and AGF averaged over 30 tests for each labeling ratio are shown in Table 1. For proper evaluation, values of $\lambda$ ranging between 0.01 and 100 were used for GTVR and LapR. In most cases, GTVM provides the most accurate classification. Note that GTVR is less sensitive to the value of $\lambda$ than LapR, and our proposed methods achieve significantly higher accuracy than LapR and HF for low labeling ratios.

\subsection{Bridge condition identification}

We next consider the bridge condition identification problem $[15,16,17]$. To validate the feasibility of indirect bridge structural health monitoring, a lab-scale bridge-vehicle dynamic system was built. Accelerometers were installed on a vehicle that travels across the bridge; acceleration signals were then collected from those accelerometers. To simulate different bridge conditions in a lab-scale bridge, masses with various weights were put on the bridge. We collected 30 acceleration signals for each of 31 mass levels, with an interval of 5 grams from 0 to 150 grams, to simulate different severity of damages, for a total of 930 acceleration signals. For more details, see [18].

The recording are represented by an 8-nearest neighbor graph, in which nodes represent recordings, and each node is connected to eight other nodes that represent the most similar recordings. The graph shift $\mathrm{A}$ are constructed as $\mathrm{A}_{i, j}=$ $\mathrm{P}_{i, j} / \sum_{i} \mathrm{P}_{i, j}$, where

$$
\mathrm{P}_{i, j}=\exp \frac{-N^{2}\left\|\mathbf{s}_{i}-\mathbf{s}_{j}\right\|_{2}}{\sum_{i, j}\left(\left\|\mathbf{s}_{i}-\mathbf{s}_{j}\right\|_{2}\right)},
$$

and $\mathbf{s}_{i}$ is a vector representation of the $i$ th recording. We randomly assigned known masses to $0.5 \%, 1 \%, 2 \%, 5 \%$ and $10 \%$ of recordings and applied the inpainting algorithms to estimate the masses for remaining nodes.

The mean square errors for estimated masses averaged over 30 tests for each labeling ratio are shown in Table 2. The proposed GTVR approach yields the lowest errors and is less sensitive than LapR to the change of the tuning parameter $\lambda$. Overall, our proposed method achieve noticeably smaller errors than Laplacian-based method for low labeling ratios.

\section{CONCLUSION}

We presented a new algorithm for signal inpainting on graphs that, unlike previous approaches, is applicable to arbitrary graphs. We formulated corresponding minimization problems and derived closed-form solutions, as well as calculated an upper bound on the resulting error. We identified the relation between our approach and previous methods via regression on graphs. Experiments on real-world datasets of online political blogs and indirect bridge structural health monitoring showed that the proposed algorithm outperforms the graph Laplacian based method. 


\section{REFERENCES}

[1] A. Sandryhaila and J. M. F. Moura, "Discrete signal processing on graphs," IEEE Trans. Signal Process., vol. 61, no. 7, pp. 1644-1656, 2013.

[2] A. Sandryhaila and J. M. F. Moura, "Discrete signal processing on graphs: Frequency analysis," IEEE Trans. Signal Process., 2013, Submitted.

[3] D. I. Shuman, S. K. Narang, P. Frossard, A. Ortega, and P. Vandergheynst, "The emerging field of signal processing on graphs: Extending high-dimensional data analysis to networks and other irregular domains," IEEE Signal Process. Mag., vol. 30, pp. 83-98, 2013.

[4] D. K. Hammond, P. Vandergheynst, and R. Gribonval, "Wavelets on graphs via spectral graph theory," Appl. Comput. Harmon. Anal., vol. 30, pp. 129-150, 2011.

[5] L. I. Rudin, Osher, and E. Fatemi, "Nonlinear total variation based noise removal algorithms," Physica D, vol. 60, no. 1-4, pp. 259-268, 1992.

[6] T. F. Chan, S. Osher, and J. Shen, "The digital TV filter and nonlinear denoising," IEEE Trans. Image Proc., vol. 10, no. 2, pp. 231-241, 2001.

[7] A. Chambolle, "An algorithm for total variation minimization and applications," J. Math. Imag. Vis., vol. 20, no. 1-2, pp. 89-97, Jan. 2004.

[8] X. Zhu, Z. Ghahramani, and J. Lafferty, "Semisupervised learning using gaussian fields and harmonic functions," in Proc. Int. Conf. Mach. Learn., 2003, pp. 912-919.

[9] M. Belkin, P. Niyogi, and P. Sindhwani, "Manifold regularization: A geometric framework for learning from labeled and unlabeled examples.," J. Machine Learn. Research, vol. 7, pp. 2399-2434, 2006.

[10] F. R. K. Chung, Spectral Graph Theory (CBMS Regional Conference Series in Mathematics, No. 92), Am. Math. Soc., 1996.

[11] D. Zhou and B. Scholkopf, "A regularization framework for learning from graph data," in Proc. Int. Conf. Mach. Learn., 2004, pp. 132-137.

[12] S. Chen, A. Sandryhaila, J. M. F. Moura, and J. Kovačević, "Adaptive graph filtering: Multiresolution classification on graphs," in Proc. IEEE Glob. Conf. Signal Information Process., Austin, TX, Dec. 2013.

[13] S. Chen, F. Cerda, P. Rizzo, J. Bielak, J. H. Garrett, and J. Kovačević, "Semi-supervised multiresolution classification using adaptive graph filtering with application to indirect bridge structural health monitoring," IEEE Trans. Signal Process., June 2013, Submitted.
[14] L. A. Adamic and N. Glance, "The political blogosphere and the 2004 u.s. election: Divided they blog," in Proc. LinkKDD, 2005, pp. 36-43.

[15] F. Cerda, J. Garrett, J. Bielak, P. Rizzo, J. A. Barrera, Z. Zhang, S. Chen, M. T. McCann, and J. Kovačević, "Indirect structural health monitoring in bridges: scale experiments," in Proc. Int. Conf. Bridge Maint., Safety Manag., Lago di Como, Italy, July 2012, pp. 346-353.

[16] F. Cerda, S. Chen, J. Bielak, J. H. Garrett, P. Rizzo, and J. Kovačević, "Indirect structural health monitoring of a simplified laboratory-scale bridge model," Int. J. Smart Struct. Syst., sp. iss. Challenge on bridge health monitoring utilizing vehicle-induced vibrations, 2013, To appear.

[17] S. Chen, F. Cerda, J. Guo, J. B. Harley, Q. Shi, P. Rizzo, J. Bielak, J. H. Garrett, and J. Kovačević, "Multiresolution classification with semi-supervised learning for indirect bridge structure health monitoring," in Proc. IEEE Int. Conf. Acoust., Speech Signal Process., Vancouver, Canada, May 2013, pp. 3412-3416.

[18] G. Lederman, Z. Wang, J. Bielak, H. Noh, J. H. Garrett, S. Chen, J. Kovačević, F. Cerda, and P. Rizzo, "Damage quantification and localization algorithms for indirect shm of bridges," in Proc. Int. Conf. Bridge Maint., Safety Manag., Shanghai, China, 2014. 